\title{
Influencia de las herramientas virtuales en el desarrollo de competencias digitales en una universidad pública del norte del Perú
}

Influence of virtual tools on the development of digital skills in a public university in northern Peru

\author{
Luis Alberto Lecca Alva a ; leca a@institutosuiza.edu.pe \\ ORCID: https://orcid.org/0000-0002-7863-616X \\ Instituto de Educación Superior Tecnológico Suiza. Perú \\ Janette Danizza Jauregui Ofracio ${ }^{\boldsymbol{b}}$; ianettejo@uncp.edu.pe \\ ORCID: https://orcid.org/0000-0002-4513-7890 \\ Universidad Nacional del Centro del Perú. Perú \\ María Guadalupe Valiente Campos de Vereau ${ }^{c} ;$ mvaliente@undc.edu.pe \\ ORCID: https://orcid.org/0000-0002-4746-7447 \\ Universidad Nacional de Cañete. Perú \\ Paola Jannet Sánchez Luján ${ }^{d}$; paola21155@gmail.com \\ ORCID: https://orcid.org/0000-0001-6281-290X \\ Unidad de Gestión Educativa Local Virú. Perú
}

Recibido: Julio / 10/2021-Revisado: Agosto /15/2021- Publicado: Septiembre /30 /2021

\section{RESUMEN}

El objetivo de este artículo fue determinar si las herramientas virtuales influyen en las competencias digitales en estudiantes de la Universidad Nacional Autónoma de Chota; ubicado en la provincia de Chota región Cajamarca, con enfoque cuantitativo, investigación del tipo básico y diseño correlacional causal. Los resultados muestran que los estudiantes están 90\% en el nivel intermedio en el uso de las herramientas virtuales, y el $75 \%$ en el nivel avanzado de las competencias digitales. Según el coeficiente de determinación R2 existe un $60,5 \%$ de influencia de las herramientas virtuales en las competencias digitales y según el coeficiente de correlación de Spearman es moderada, positiva y muy significativa al 0,778. Concluyendo que; las herramientas virtuales permiten ampliar los conocimientos académicos de manera virtual a través de grupos colaborativos, aumentando las competencias digitales en los estudiantes de la Universidad Nacional Autónoma de Chota.

Palabras claves: Herramientas virtuales; competencias digitales; educación universitaria.

\section{ABSTRACT}

The objective of this article was to determine if virtual tools influence digital skills in students of the National Autonomous University of Chota; located in the province of Chota Cajamarca region, with a quantitative approach, research of the basic type and causal correlational design. The results show that the students are $90 \%$ at the intermediate level in the use of virtual tools, and $75 \%$ at the advanced level of digital skills. According to the determination coefficient $R 2$ there is a $45.5 \%$ influence of virtual tools on digital skills and according to the Spearman correlation coefficient it is moderate, positive and highly significant at 0.778. Concluding that; Virtual tools allow to expand academic knowledge in a virtual way through collaborative groups, increase digital skills in students of the National Autonomous University of Chota.

Keywords: Virtual tools; digital skills; University education. 


\section{Introducción}

A nivel internacional se observa que merced al advenimiento del covid-19 los entornos virtuales se están convirtiendo en una herramienta alternativa para la enseñanza y aprendizaje en el nivel universitario, entre ellos las herramientas virtuales nos han estado sirviendo para fortalecer las competencias digitales de los estudiantes de la Universidad Nacional Autónoma de Chota.

Teniendo como referente a la UNESCO (2018), se ha reconocido el uso intensivo de las tecnologías de la información y comunicación en todo proceso, entre ellos los relacionados al trabajo universitario.

De igual manera, según el Informe Anual de Internet de Cisco (2020), con prospectiva al 2023 nos arroja los siguientes resultados, el 66\% de la población mundial tendrá acceso a Internet. Habrá 29,3 mil millones de dispositivos conectados ( $50 \%$ de las conexiones) y las velocidades $5 \mathrm{G}$ alcanzarán 13 veces la conexión móvil promedio.

En esta investigación se han considerado las teorías del ámbito psicológico y pedagógico que sustentan el aprendizaje han ido evolucionado, desde las conductistas hasta las cognitivas respaldadas en el uso intensivo de las tecnologías de la información y comunicaciones, y con ello los modelos formativos sustentados en las tecnologías web.

Para fundamentar la investigación, se tomó como antecedentes los trabajos realizados por: Serrano et. al. (2019) quien desarrolla la investigación: La información de estudiantes universitarios en entornos de aprendizaje individuales, quien concluye que los futuros profesionales son conscientes de la necesidad de comparar la información recibida y aplicar el pensamiento crítico, cuestionar la información recibida del exterior y respaldar sus expectativas con el uso de herramientas tecnológicas. 
Luego González et al. (2019) realizó la investigación: El aprendizaje colaborativo a través de herramientas digitales en un entorno universitario, quien concluye que los métodos de trabajo en equipo son particularmente importantes en contextos educativos. Integrando todos los aspectos, trabajo colaborativo y competencias digitales y desarrollaron actividades teóricas y prácticas en diversas materias de la titulación.

Zapana (2020) en su investigación: Competencias digitales y motivación académica en la actitud hacia la investigación científica en la Universidad San Martin de Porres Lima, 2019, quien concluye que, con buena motivación académica, la probabilidad de la actitud hacia la investigación científica es media y muy significativa.

Vidal (2019) en su investigación: Aplicación de un módulo de autoaprendizaje sobre el uso de la web 2.0 para el mejoramiento de las habilidades en el ámbito laboral de los estudiantes de Doctorado, quien concluye que existe un impacto positivo en la implementación del trabajo en equipo, puede comunicarse a través de las herramientas propuestas en el módulo y poder realizarlas con normalidad.

Larico (2018) en su investigación: El aula virtual y el aprendizaje del algoritmo en los estudiantes de la escuela profesional de ingeniería de sistemas e informática, la investigación concluye que el aula virtual a la cual acceden los estudiantes tiene un impacto muy significativo en el aprendizaje de ellos en los cursos de algoritmos.

Respecto a las teorías cognitivas sitúa al estudiante en una posición de autonomía para obtener el aprendizaje por iniciativa propia, motivándolo a ser un individuo con decisión para aprender y propiciar el desarrollo de habilidades y destrezas (Sosa, et al., 2015). Esta teoría argumenta sus actividades internas para remodelar, reorganizar y reconstruir programas y modelos mentales en el proceso de aprendizaje, por lo que es el estudiante quien construye e interpreta la realidad. 
También se ha tenido en cuenta la teoría del conectivismo propuesta por Siemens (2004), quien considera al conectivismo como la aplicación de principios de trabajo en red para definir procesos de conocimiento y aprendizaje. En esa óptica el conocimiento se define como un patrón de relación específico, mientras que el aprendizaje se define como la creación de nuevas conexiones y patrones y la capacidad de resolver redes o patrones existentes.

En esa línea, el conexionismo aborda los principios del aprendizaje en varios niveles: células nerviosas biológicas, conceptos e influencias sociales externas (Zapata, 2015).

En ese sentido, con el desarrollo e integración de herramientas de software social, el sujeto puede interactuar con otros de una manera como el crea que sea necesaria, siendo capaces de transformar culturas, las redes sociales permiten a las personas estar conectadas en tiempo real y compartir sus conocimientos a nivel mundial generando un aprendizaje colaborativo. (Gallar et al.,2015).

Según Real (2019) describe que las herramientas virtuales mencionadas como programas y/o plataformas que pueden ayudar a los maestros a preparar su propio contenido digital, y también es considerado como medios digitales y materiales educativos, mediante herramientas que pueden ser dispositivos móviles en computadoras o internet.

Por ello, los maestros deben adquirir esta habilidad porque es uno de los sellos distintivos de su perfil de graduado actual, debido a que las TIC tienen un gran potencial para desarrollar la inteligencia de los estudiantes, necesitan usar herramientas digitales para alentar a los estudiantes a aprender de manera independiente y significativa.

Para Avidon (2020) reconoce a las dimensiones de las herramientas virtuales como las que puede utilizarlos en la docencia tales como las herramientas de almacenamiento de información, como las de compartir o almacenar documentos 
temáticos en la nube, en Google Drive, One drive, Mega, Dropbox, etc., las herramientas de colaboración como Moodle, Google Classroom y Chamillo; las herramientas de comunicación como el aula y guía las actividades de enseñanza a través de aplicaciones como Zoom, Google meet, Skype y otras. Herramientas utilizadas para crear contenido, donde se utiliza para construir contenidos educativos para compartir información en otros espacios, plataformas, especialmente otras redes, utilizando Kahoot, Excel, Learning, Powtoon, entre otros aspectos, y finalmente las herramientas de evaluación que permiten la valoración normal de las materias mediante el uso de programas como Socrative para medir el nivel de aprendizaje alcanzado en una materia determinada.

Respecto a las competencias digitales, según Viñals y Cuenca (2016) mencionan que el continuo desarrollo de la tecnología digital e internet nos permite vivir en un entorno digital conectado. El método de aprendizaje y enseñanza cambió. Por su lado la Comisión Europea (2007) define a la capacidad digital como el uso crítico y seguro de las tecnologías de la sociedad de la información en el trabajo, el ocio y la comunicación.

Por su lado Ferrari (2013) precisa que, las discusiones sobre alfabetización generalmente se enfocan en decodificar y codificar o leer y escribir, pero aquí nos referimos a las habilidades digitales como un conjunto de conocimientos, habilidades y actitudes. Luego Vargas (2019) refiere que las competencias digitales son las habilidades y competencias que los profesores universitarios cultivan en su labor docente y los estudiantes en el proceso de aprendizaje.

Según Flores y Garrido (2019) señalan que las competencias digitales son un mecanismo para enfrentar los desafíos sociales actuales conlleva cambios profundos que van más allá del uso instrumental de la tecnología. Los profesores tienen buenas habilidades teóricas, pero necesitan integrar la tecnología y las habilidades de enseñanza para conectarse con las necesidades de la sociedad actual. 
Por su lado Suarez y Orgaz (2019) tomaron como fundamento los estándares desarrollados por la International Society for Technology in Education (ISTE) especialmente del proyecto NETS*S 2007 (ISTE, 2008) y propuso las siguientes dimensiones: Acceso y uso de la información, la que permite determinar la utilización de las herramientas digitales para obtener, evaluar y utilizar la información necesaria. Alfabetización tecnológica donde los estudiantes muestran un conocimiento completo de los conceptos, sistemas y funciones de las tecnologías de la información y la comunicación.

La Comunicación y colaboración, en donde los estudiantes pueden utilizar los recursos digitales y el entorno para comunicarse y realizar un trabajo colaborativo, e incluso pueden realizar colaboraciones a distancia para ayudar a las personas a aprender y promover el aprendizaje de los demás. Asimismo, la Ciudadanía digital, en la que además de ejercer un comportamiento legal y ético, los estudiantes también comprenden cuestiones humanas, culturales y sociales relacionadas con las tecnologías de la información y la comunicación. Por último, la Creatividad e innovación, en la que, los estudiantes muestran ideas creativas que pueden utilizar las TIC para generar conocimiento y desarrollar productos y procesos innovadores.

De ello se formuló la pregunta de investigación: ¿Cuál es la influencia que existe entre las herramientas virtuales en las competencias digitales en estudiantes de la Universidad Nacional Autónoma de Chota, 2021?

En el presente artículo se planteó el objetivo: Determinar si las herramientas virtuales influyen en las competencias digitales en estudiantes de la Universidad Nacional Autónoma de Chota, 2021.

\section{Metodología}

El enfoque de investigación es cuantitativo, la investigación es del tipo aplicada, del nivel correlacional, y diseño correlacional causal, de corte transversal, debido a la finalidad de las variables, en donde se observa si la primera o 
independiente influye en la segunda o dependiente en un contexto en particular (Hernández et al., 2010).

Los instrumentos fueron aplicados a 174 estudiantes de las 5 carreras profesionales de las 4 facultades de la Universidad Nacional Autónoma de Chota, elegidos por conveniencia de acuerdo con la accesibilidad y disposición de tiempo de los estudiantes.

Para ambas variables se utilizó el cuestionario, cuyas preguntas fueron formuladas según los indicadores de la variable debidamente sistematizadas, distribuidas en las dimensiones de los cuales se obtuvo un coeficiente de 0.987 en la confiabilidad y para la validación se realizó por la de constructo confirmatoria igual a 0,895 .

Además, en ambos casos se tuvo en cuenta el criterio del consentimiento informado al momento de aplicar el instrumento y la base de datos de su historial académico, de cada uno de los integrantes de la muestra de estudio (Oseda, Ruiz, Hurtado, y Añaños 2020).

\section{$\underline{\text { Resultados }}$}

Se presentan los resultados obtenidos referente a las variables Herramientas virtuales y Competencias digitales recolectados por medio de los instrumentos.

\section{Tabla 1}

Porcentajes de resultados obtenidos de la variable Herramientas virtuales y Competencias digitales.

\begin{tabular}{lcccc}
\hline Variable & \multicolumn{2}{c}{ Herramientas virtuales } & \multicolumn{2}{c}{ Competencias digitales } \\
\hline & $\mathrm{F}$ & $\%$ & $\mathrm{f}$ & $\%$ \\
Avanzado & 16 & 9 & 122 & 70 \\
Intermedio & 158 & 91 & 52 & 30 \\
Básico & 0 & 0 & 0 & 0 \\
Total & 174 & 100 & 174 & 100 \\
\hline
\end{tabular}

Nota. Datos obtenidos de la base de datos de los investigadores. 
Los estudiantes se encuentran en su mayoría en el nivel intermedio y en el nivel avanzado $100 \%$ para la primera variable y $100 \%$ para la segunda variable, y ninguno se encuentra en el nivel básico.

\section{Tabla 2}

Relación entre las variables herramientas virtuales y competencias digitales

\begin{tabular}{lllc|c}
\hline & & $\begin{array}{c}\text { Herramientas } \\
\text { virtuales }\end{array}$ & $\begin{array}{c}\text { Competencias } \\
\text { digitales }\end{array}$ \\
\hline Herramientas virtuales & Correlación de Spearman & 1 &, $778^{* *}$ \\
\cline { 2 - 4 } & Sig. (bilateral) & 174 &, 000 \\
\cline { 2 - 4 } & $\mathrm{N}$ &, $778^{* *}$ & 174 \\
\hline Competencias digitales & Correlación de Spearman &, 000 & 1 \\
\cline { 2 - 4 } & Sig. (bilateral) & 174 & 174 \\
\cline { 2 - 4 } & $\mathrm{N}$ & & \\
\hline
\end{tabular}

**. La correlación es significativa en el nivel 0,01 (bilateral).

Nota. Datos obtenidos de la base de datos de los investigadores.

La correlación que existe entre las variables, según Spearman es de 0,778** positiva fuerte y muy significativa; con un p-valor: 0,00<0,05 evidenciando la influencia de la primera variable independiente en la segunda variable dependiente.

\section{Tabla 3}

Datos alcanzados por el coeficiente de determinación R2

\begin{tabular}{lc|ccc}
\hline Modelo & R & R cuadrado & $\begin{array}{c}\text { R cuadrado } \\
\text { ajustado }\end{array}$ & $\begin{array}{c}\text { Error estándar de } \\
\text { la estimación }\end{array}$ \\
\hline 1 &, $778^{\mathrm{a}}$ &, 605 &, 582 & 8,687 \\
\hline
\end{tabular}

a. Predictores: (Constante), Competencias digitales

El coeficiente de determinación donde el $60,5 \%$ de la variabilidad de Competencias digitales se expresa por la influencia de Herramientas virtuales. 


\section{Tabla 4}

Relación entre las variables Herramientas virtuales y dimensiones de la segunda variable por tener normalidad.

\begin{tabular}{ll|c|c|c}
\hline & \multicolumn{1}{c}{$\begin{array}{c}\text { Acceso y uso de } \\
\text { la información }\end{array}$} & $\begin{array}{c}\text { Ciudadanía } \\
\text { digital }\end{array}$ & $\begin{array}{c}\text { Creatividad e } \\
\text { innovación }\end{array}$ \\
\hline $\begin{array}{l}\text { Herramientas } \\
\text { virtuales }\end{array}$ & $\begin{array}{l}\text { Correlación de } \\
\text { Spearman }\end{array}$ &, $566^{* *}$ &, $496^{* *}$ &, $648^{* *}$ \\
\cline { 2 - 5 } & Sig. (bilateral) &, 000 &, 000 &, 000 \\
\hline & $\mathrm{N}$ & 174 & 174 & 174 \\
\hline
\end{tabular}

Nota. Datos obtenidos de la base de datos de los investigadores.

Se observa la correlación altamente significativa directa, positiva y moderada entre herramientas virtuales con las dimensiones Acceso y uso de la información, Ciudadanía digital, Creatividad e innovación, según Spearman es de; 0,566**, $r=, 496^{\star \star}, r=, 648^{\star *}$, $p$-valor: $0,00<0,05$ respectivamente.

\section{Tabla 5}

Relación entre las variables Herramientas virtuales y las dimensiones de la segunda variable por no tener normalidad

\begin{tabular}{lllc|c|c}
\hline & & $\begin{array}{c}\text { Herramientas } \\
\text { virtuales }\end{array}$ & $\begin{array}{c}\text { Alfabetización } \\
\text { tecnológica }\end{array}$ & $\begin{array}{c}\text { Comunicación } \\
\text { y colaboración }\end{array}$ \\
\hline $\begin{array}{l}\text { Rho de } \\
\text { Spearman }\end{array}$ & $\begin{array}{l}\text { Herramientas } \\
\text { virtuales }\end{array}$ & $\begin{array}{l}\text { Coeficiente de } \\
\text { correlación }\end{array}$ & 1,000 &, $498^{* *}$ &, $558^{* *}$ \\
\cline { 2 - 6 } & & Sig. (bilateral) & $\cdot$ &, 000 &, 000 \\
\cline { 2 - 6 } & $\mathrm{N}$ & 174 & 174 & 174 \\
\hline
\end{tabular}

$$
\text { **. La correlación es significativa en el nivel 0,01 (bilateral). }
$$

Nota. Datos obtenidos de la base de datos de los investigadores.

Se observa la correlación altamente significativa directa, positiva y moderada entre herramientas virtuales con las dimensiones Alfabetización tecnológica, Comunicación y colaboración, según Spearman es de; Rho $=0,498^{* *}$, Rho $=0,558^{* *}$ p-valor: 0,00<0,05 respectivamente. 


\section{Discusión}

Después del procesamiento de datos se ha realizado el análisis y la comparación de estos a través de la triangulación, con los antecedentes y teorías, dando respuesta a los objetivos planificados en la investigación. Se observó que consideran que en la variable herramientas virtuales, el $90 \%$ se encuentran en el nivel intermedio, en competencias digitales el $75 \%$ manifestaron estar en un nivel avanzado.

Para reforzar la investigación Zapana (2020) menciona que las competencias digitales tienen influencia significativa en la actitud del estudiante hacia la investigación científica en estos entornos virtuales. Es por ello, que es necesario considerar el uso de las nuevas tecnologías informáticas hacer uso intensivo de todos los recursos para la adquisición de conocimientos que lleven a clarificar diversos aspectos considerados en los planes de estudios curriculares.

Sin duda alguna, la influencia de la variable independiente hacia la variable dependiente, según el coeficiente de correlación de Spearman es de $0,778^{\star \star}$ existe una correlación positiva, moderada y muy significativa; y según el coeficiente de determinación donde el $60,5 \%$ de la variabilidad de las competencias digitales se expresa por la influencia de las herramientas virtuales. Por lo tanto, al utilizar las herramientas virtuales aumenta también en la misma proporción las competencias digitales en los estudiantes universitarios de este claustro universitario. Estos estudios se ven reforzados con los hallazgos de Saberbein (2018) que tiene coincidencia total con los resultados de la presente investigación al dar a conocer la relación encontrada entre sus variables fue de 0,639 ( $p$-valor: 0,00<0,01) según el parámetro estadístico de la Rho de Spearman existiendo una relación positiva y altamente significativa.

Las teorías psicológicas y pedagógicas existentes apoyan de manera positiva y favorable el estudio al coincidir con lo planteado y los hallazgos que sustentan el 
aprendizaje que en función al tiempo han ido cambiando, dando lugar el énfasis de las teorías conductistas a las teorías cognitivas, que es la que más apoyan las tecnologías de la información y comunicación, y también los modelos formativos sustentados en las tecnologías web.

Desde una perspectiva cognitivista esta íntimamente asociada a la teoría de Vygotsky (1978) la cual coincide plenamente con lo planteado y encontrado para llegar a conclusiones confirmando que el aprendizaje significativo se promueve con la interacción de los estudiantes universitarios, o sea le da la importancia debida a la formación personal y académica. Respecto al soporte o andamiaje pedagógico para promover el desarrollo cognitivo de los estudiantes universitarios en el proceso de aprendizaje, en nuestro caso en los entornos virtuales periodo covid19.

También Piaget (1995) se centra en la construcción del conocimiento a partir de la interacción con el entorno inmediato, y es uno de los principales impulsores del cognitivismo y estudia principalmente la epistemología genética del desarrollo cognitivo y la formación del conocimiento lo que favorece al estudiante universitario en el desarrollo formativo - integral de nuestro contexto.

La teoría conectivista de Siemens (2004) hace énfasis al tipo de teoría del aprendizaje que en la era digital considera que el aprendizaje significativo es un proceso que tiene lugar en cualquier contexto, en un entorno disperso, ambiguo y en constante cambio. Definitivamente el conexionismo aborda los principios fundamentales del aprendizaje en varios niveles como son las células nerviosas biológicas, los conceptos e influencias sociales externas (Zapata, 2015).

No hay lugar en el mundo hoy en día donde no se esté usando las tecnologías informáticas en el aprendizaje de nuestros estudiantes, cualquiera fuese el nivel educativo, por lo que urge retomar y mejorar los procesos y por ende los resultados a corto y mediano plazo, El futuro ya es el presente, la educación virtual llegó y no se va ir muy pronto, tenemos mucho camino que recorrer y en ese camino debemos mejorar la dotación científico tecnológica en nuestras universidades públicas del país. 


\section{Conclusiones}

- Se ha determinado que las herramientas virtuales han influido significativamente en las competencias digitales en estudiantes de la Universidad Nacional Autónoma de Chota, al ampliar sus conocimientos académicos de manera virtual a través de grupos colaborativos reconociendo el $90 \%$ en nivel intermedio, también los estudiantes están utilizando el conocimiento de las TIC para lograr un desarrollo en lo académico, personal, social y cultural en $75 \%$ nivel avanzado.

- La influencia de las herramientas virtuales en las competencias digitales según el coeficiente de determinación $\mathrm{R}^{2}$ es de $60,5 \%$ variabilidad de competencias digitales expresada por la influencia de herramientas virtuales. El coeficiente de la correlación de Spearman es $0,778^{* *}$ positiva, fuerte y significativa con p-valor menor a 0,05 entre la variable independiente y dependiente.

\section{Referencias}

Avidon, F. M. (2020). Uso de Entornos Virtuales en la Gestión Áulica del Servicio Educativo No Presencial en las Instituciones Educativas de Educación Básica Regular en la Región San Martín, en tiempos de Pandemia. México DF.

CISCO (2020). Informe anual de Internet de cisco 2018-2023. 21 feb. 2020. San José, California: https://universoabierto.org/2020/02/21/informe-anual-de-cisco-sobreinternet-2018-2023/

Comisión Europea (2007). Competencias clave para el aprendizaje permanente. Un marco de referencia europeo. Unión Europea.

Ferrari, A. (2013). DIGCOMP: A Framework for Developing and Understanding Digital. Competence in Europe. Seville, Spain: Institute for Prospective Technological Studies (IPTS).

Flores, D. K., y Garrido, J. E. (2019). Competencias digitales para los nuevos escenarios de aprendizaje en el contexto universitario. Revista Scientific, 4(14), 44-61, e-ISSN: 2542-2987. https://doi.org/10.29394/Scientific.issn.2542-2987.2019.4.14.2.44-61

Gallar P. Y., Rodríguez, I. E., y Barrios, E. A. (2015). La mediación con las TICS en la enseñanza-aprendizaje de la Educación Superior. Didasc@lia: Didáctica Educ [en línea]. 2015 Dic [citado 24/10/2018]; VI (6):155-164. https://www.researchgate.net/publication/328918192_LA_MEDIACION_CON_LA 


\section{S_TIC_EN_EL_PROCESO_DE_ENSENANZA- APRENDIZAJE_DE_LA_EDUCACION_SUPERIOR}

González, M. D., Abad, E. y Belmonte, L. J. (2019). Meaningful learning in the development of digital skills. Trend análisis. Análisis de tendencias. International Journal of Educational Research and Innovation (IJERI), 14, 91-110 ISSN: 2386-4303 DOI https://doi.org/10.46661/ijeri.4741

Hernández, R., Fernández, C., y Batista, L. (2010). Metodología de investigación. Ciudad de México, México: Mc Graw Hill.

Larico, G. R. (2018). El aula virtual y el aprendizaje del algoritmo en los estudiantes de la escuela profesional de ingeniería de sistemas e informática de la Universidad Nacional Amazónica de Madre de Dios, región Madre de Dios - 2017. [Doctor en Ciencias de la Educación. Enrique Guzmán y Valle]. https://repositorio.une.edu.pe/handle/UNE/2607

Oseda, D., Ruiz, J. O., Hurtado, D. R., \& Añaños, M. A. (2020). Clima social familiar y rendimiento académico en estudiantes de una universidad pública de Lima. Revista Conrado, 16(77), 26-31.

Pedró, F. (2021) La transformación digital durante la pandemia de la covid-19 y los efectos sobre la docencia. https://www.iesalc.unesco.org/2021/01/24/la-transformaciondigital-durante-la-pandemia-de-la-covid-19-y-los-efectos-sobre-la-docencia/ director del Instituto Internacional de la UNESCO para la Educación Superior en América Latina y el Caribe (IESALC), durante su intervención, el 21 de enero de 2021, en el foro "La Educación Superior en Nicaragua y el Impacto del COVID-19", organizado por la Universidad Nacional Autónoma de Nicaragua (UNAN).

Piaget, J. (1995). La teoría de Piaget, en: Infancia y Aprendizaje. Barcelona. España.

Real, C. (2019). Materiales Didácticos Digitales: un recurso innovador en la docencia del siglo XXI. 3C TIC. Cuadernos de desarrollo aplicados a las TIC, 8(2), 12-27. doi: http://dx.doi.org/10.17993/3ctic.2019.82.12-27

Saberbein M., J. C. (2018). Enseñanza estratégica virtual, aprendizaje colaborativo y formación de tutores en Orientación Vocacional en la Facultad de Tecnología de la Universidad Nacional de Educación, 2016. [Enrique Guzmán y Valle. Doctor en Psicología $\quad$ Educacional y $\quad$ Tutorial] https://repositorio.une.edu.pe/bitstream/handle/UNE/2163/TD\%20PE\%201912\%20 S1\%20-\%20Saberbein\%20Mu\%c3\%b1oz.pdf?sequence=1\&isAllowed=y

Serrano, J. L., Carrera, X., Brescó, B. E., y Suarez, G. C. (2019). Tratamiento crítico de la información de estudiantes universitarios desde los entornos personales de $\begin{array}{lllll}\text { aprendizaje. } & \text { Educação } & \text { Pesquisa, } & 1-21 .\end{array}$ https://www.scielo.br/scielo.php?pid=S151797022019000100552\&script=sci_arttext

Siemens, G. (2004). Conectivismo: Una teoría de aprendizaje para la era digital. Traducción: Leal F., D. E. (2007) (diego@diegoleal.org), febrero 7, 2007. 
Sosa, R., García, S., Sánchez, A., Moreno, A., y Reinoso, A. (2015). B-Learning y Teoría del Aprendizaje Constructivista en las Disciplinas Informáticas: Un esquema de ejemplo a aplicar. Recent Res Dev Lear Technol. 2015 [citado 24/10/2018]1-6. http://1.asset.soup.io/asset/2112/4657_4fa5.pdf

Suarez, C., y Orgaz, F. (2019). Perfil digital y expectativas profesionales sobre tecnología en estudiantes universitarios. Revistas 40. https://www.revistaespacios.com/a19v40n21/19402129.html

UNESCO. (2018). Rendir cuentas en el ámbito de la educación: cumplir nuestro compromiso. Paris: UNESCO.

Vargas, G. (2019). Competencias Digitales y su Integración con Herramientas Tecnológicas en Educación Superior. Revista "Cuadernos". Vol. 60(1). 2019: 88-94. ISSN 15626776. http://www.scielo.org.bo/pdf/chc/v60n1/v60n1_a13.pdf

Vidal, M. L. (2019). Aplicación de un módulo de autoaprendizaje sobre el uso de la web 2.0 para el mejoramiento de las habilidades en el ámbito laboral de los estudiantes de Doctorado de la Escuela de Posgrado de la Universidad Nacional de Educación Enrique Guzmán y Valle 2015 - 2018. [Universidad Nacional de Educación Enrique Guzmán y Valle. Tesis doctoral]. https://repositorio.une.edu.pe/handle/UNE/3042

Viñals, K. y Cuenca, L. (2016). El rol del docente en la era digital. Revista Interuniversitaria de Formación del Profesorado, 30(2), 103-114. [Universidad de Zaragoza]. https://www.redalyc.org/jatsRepo/274/27447325008/html/index.html.

Vygotsky, L. S. (1978). Mind in Society. Cambridge, MA: Harvard University Pres.

Zapana, D. (2020). Competencias digitales y motivación académica en la actitud hacia la investigación científica en la Universidad San Martin de Porres Lima, 2019. [Universidad San Martin de Porres. Tesis de Doctorado]. Lima. Perú. Recuperado de http://renati.sunedu.gob.pe/handle/sunedu/897420.

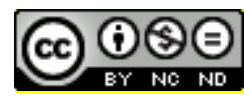

Influencia de las herramientas virtuales en el desarrollo de competencias digitales en una universidad pública del norte del Perú (Luis Alberto Lecca - Alva) Por Revista $\underline{\text { Sendas se encuentra bajo una Licencia Creative Commons-No Comercial-Sin Derivadas }}$ 3.0 Uported. 